\title{
SPEECH ENABLED INTERACTIVE VOICE RESPONSE SYSTEM
}

\author{
Mahammad Rafi ${ }^{1}$, Khan Sohail Ahmed ${ }^{2}$, Shaikh Huda ${ }^{3}$, Lutful Islam ${ }^{4}$ \\ ${ }^{\text {I}}$ Student,Computer, M.H SabooSiddik College of Engineering, Maharashtra, India \\ ${ }^{2}$ Student,Computer, M.H SabooSiddik College of Engineering, Maharashtra, India \\ ${ }^{3}$ Student,Computer, M.H SabooSiddik College of Engineering, Maharashtra, India \\ ${ }^{4}$ Assistant Professor,Computer, M.H SabooSiddik College of Engineering, Maharashtra, India
}

\begin{abstract}
In this paper we propose a new product inwhich speech is used to interact with computers. Speech is a man's most powerful form of communication. The user will be able to give various voice commands to the system, which the system will recognize and execute tasks based on the input command. This system will provide another form of input (apart from mouse and keyboard) for daily users. It will also be of great assistance to physically challenged users. The user will be able to perform all the operations using his voice as input which he is able to perform normally using mouse and keyboard. The system however requires a little bit training from the user, so that the system will understand the user better. The need of training is due to the fact that every person has different voice. Also the voice of women is totally distinct from men. More training will result in faster and accurate response. Extensive experiments are conducted in order to check the accuracy and efficiency of the system, and the results show that our system is reliable and achieves better accuracy and efficiency than previous systems.
\end{abstract}

KeyWords: Speech Technology, Voice Response System, Voice User Interface, Voice Recognition.

\section{INTRODUCTION}

Speech is man's most effective and natural mode of communication. But we cannot use it for man-machine communication. The last few years has seen stunning growth of speech technology with speech based applications ranging from dictation packages to hardware control seen in cockpits of pilots. Speech technology is on the verge of widespread application. But still, has not been able to make a significant mark in computer application because of its several drawbacks.

Speech Enabled Interactive Voice Response System provides a natural and social-style interaction mode by which humans can communicate with computer. This interaction mechanism is also very important for a broad range of users such as physically impaired users, users working in hostile environments. Several research endeavors have been assigned to endow the humans with verbal interaction as a high-level faculty with computers. In this project, we investigate a tested platform that can be deployed to enable human-computer verbal interaction. The proposed approach encompasses a design pattern-based user interface and a user-independent automatic speech recognizer with a modified grammar module in the context of human-robot interaction. The user interface is used to simulate computers to respond (perform specific requested task) based on the voice commands given.

Any two interacting parties rely heavily on the communication medium between them. The better the communication interface between them, the better will be the communication and faster will be the transmission of information. With the advent of PCs, keyboards and mouse have been the most preferred interfaces to computers. But, the keyboard or the mouse is certainly not the fastest way of transferring data to the machine. This gives rise to considering whether speech, being the most natural form of communication, can be considered as one form of dialog between man and machine.

The 'voice user interface' is evolving into a standard means of communication between humans and technology and is having a profound influence on the way people live. Speech technology has seen tremendous development during the last few years with several speech-based applications prominently used in dictation, interactive voice response systems, security,games based on interactive voice commands and so on. But speech has proved to be an inefficient input mechanism with respect to several types of computer applications. The field of computer games has been one such domain where it has certainly failed to deliver although speech has been found extremely beneficial in certain circumstances.

\section{EXISTING SYSTEM}

The existing system is not a voice controlled system. Work is carried out in current system manually using keyboard \& mouse, which makes it difficult for the physically impaired people to use the system without assistance of other individual or group of individuals.

Such people cannot use computer without the assistance of other people. This reduces their efficiency to perform tasks as well as they continuously require helping aid which is not always possible to be provided. Provision of helping aid also increases the institutions expenses.

If the User/Student has to access a particular Microsoft product for example Microsoft Word \& he/she has to perform some typing work, he/she may require a typist to accomplish this task since he/she cannot perform the work manually using the keyboard or mouse due to physical disability. Also the person aiding the student should be 
quick \& efficient to understand as well as provide proper training.

Hence the manual process with assistance is inefficient \& also increases expenses. Also there is wastage of time in training the physically challenged person to communicate \& also when the student communicates with the person assisting him or her.

\section{PROPOSED SYSTEM}

We will try to include the entire above feature suggested for the proposed system. We will try to make the system user friendly avoiding the complexity of the work done. We will try to take minimum required inputs from the user \& will give the desired output in the system.

The Student/user can begin to use it without prior programming knowledge or computer skills.

As the programming language we have decided for Visual Basic.NET because as per the user they want it very user friendly which could be done in Visual Basic.NET easily. Also we are going to use Microsoft SAPI SDK.

\section{DESIGN IMPLEMENTATION}

Speech Enabled Interactive Voice Response System takes user's voice as input.

Brief Summary of System Flowchart:

- User gives voice command to system

- It is recognized by intermediate tool SAPI.

- The SAPI converts analog signal into its digital representation.

- System will search in its Grammar File for matching respective command.

- Based on the match it will execute operation (For example: User Speaks “Open MS Word”. The System will start MS Word.).

The flow chart of system is shown below.

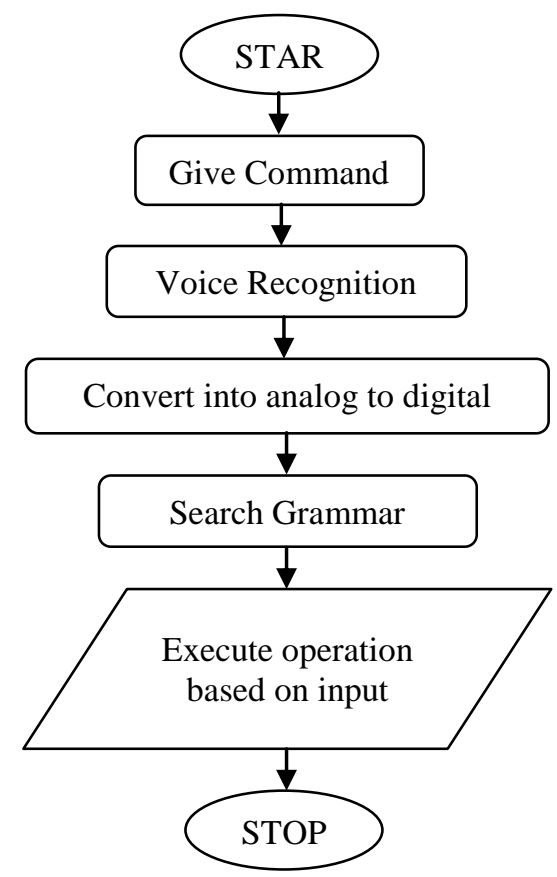

Figure 1: Flowchart of SEIVRS

\section{CONCLUSIONS}

Thus we have successfully implemented the various software and different kind of languages to develop the SEIVRS.

It has various advantages and disadvantages which has been explained above. We have tried our level best to make the software user friendly and specific to match the exact needs of our client.

Currently System is only limited to use of keyboard \& mouse with assistance to the User. It can be made compatible with voice commands used in various Windows platforms using Speech Application Programming Interface (SAPI) or .NET's system. Speech managed name space.

For developing applications on the Windows desktop operating system, you can use client-side APIs that you can use to enhance your Windows applications, either using native code or the Speech Application Programming Interface (SAPI) or .NET's system. Speech managed name space.

Also the existing system is limited to key products like Microsoft Windows, Microsoft Office, and Microsoft Unified Communications

\section{ACKNOWLEDGEMENT}

No project can be completed without the support of a lot of people. When we are concluding our project synopsis work by submitting this report, we reflect upon all the times when we needed support in various forms and were lucky enough to receive it.

A special mention here to Prof. Z. A. USMANI (H.O.D., Computer Engineering Department, and MHSSCOE) for his valuable support. We are also thankful to all staff members of Computer Department, without whom the completion of this report would have been impossible.

This entire journey would not have been possible without the efforts put in by our guides, ER. LUTFUL ISLAM. They have been a constant source of encouragement and guidance through the entire semester.

This acknowledgment would indeed be incomplete without rendering our sincere gratitude to our family and wellwishers. They have always been a pillar of strength and support in our past and current endeavors

\section{REFERENCES}

[1].Fundamentals of Speech Recognition 1st Edition by Lawrence Rabiner (Author), Biing-Hwang Juang (Author).

[2].Speech based system. N.K. Vela rum Mohan Ram (Author).

[3].http://www.phon.ucl.ac.uk/courses/spsci/compmeth/spee ch/recognition.html 


\section{BIOGRAPHIES}

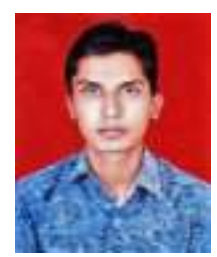

Name: Mr. Mahammad Rafi

Designation: Student

Department: Computer Engineering

Qualifications: B.E.(comp)Pursuing

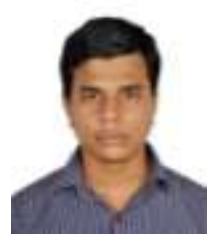

Name: Mr. Khan Sohail

Designation: Student

Department: Computer Engineering

Qualifications: B.E.(comp)Pursuing

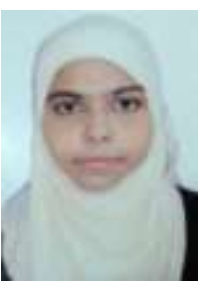

Name: Ms. Shaikh Huda

Designation: Student

Department: Computer Engineering

Qualifications: B.E.(comp)Pursuing

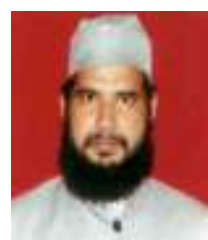

Name: Mr. Md. Lutful Islam

Designation: Assistant Professor

Department: Computer Engineering

Qualifications: M.C.A, M. Tech(Comp) 